\title{
Asymptotical stabilization of the nonlinear upper triangular fractional-order systems
}

\author{
Yige Zhao ${ }^{1 *}$ (D), Yibing Sun', Yilin Wang ${ }^{2}$ and Zhanbing Bai ${ }^{3}$
}

\author{
*Correspondence: \\ zhaoeager@126.com \\ 'School of Mathematical Sciences, \\ University of Jinan, Jinan, P.R. China \\ Full list of author information is \\ available at the end of the article
}

\begin{abstract}
This paper introduces a simple method of the design of the output feedback stabilizing controller (OFSC) for the nonlinear upper triangular fractional-order systems (NUTFOS). The OFSC which makes the closed-loop system asymptotically stable is given based on the fractional indirect Lyapunov method and the static gain control method. Furthermore, an algorithm is established to design OFSC for the NUTFOS. Finally, an example is presented to verify the validity of the proposed method.

Keywords: Nonlinear fractional-order system; Upper triangular system; Output feedback; Stabilizing controller; Fractional indirect Lyapunov method
\end{abstract}

\section{Introduction}

Fractional-order systems (FOS) have received a great deal of attention from mathematicians, physicists, chemists, biologists, and so on [1-14]. It has been the huge development of the theory and the applications in many fields, especially in control. The feedback control design problem is a hot topic for nonlinear fractional-order systems, such as linear matrix inequality (LMI) methods [15-21], adaptive backstepping control scheme [22-24], the static gain control method [25-27], etc.

Linear matrix inequality (LMI) methods have been used to discuss the feedback control design problem of fractional-order systems; see [17-21]. The existence conditions and design methods of the state feedback controller, static output feedback controller, and observer-based controller for asymptotically stabilizing such uncertain linear FOS were derived in [17]. The fuzzy output feedback stabilization for uncertain FOS was considered in [18]. The output feedback normalization and stabilization for singular FOS were investigated in [19]. Based on a fractional-order indirect Lyapunov method, a new singular system approach, and linear matrix equality (LMI) methods, the problem of output feedback sliding mode control for nonlinear FOS was studied in [20]. A robust fixed-order dynamic output feedback controller for uncertain linear time-invariant FOS was designed in [21].

Adaptive backstepping control scheme has been also used to considered the feedback control design problem of FOS; see [22-24]. Mittag-Leffler stability of nonlinear FOS was solved via fractional-order backstepping in [22]. A novel fractional order adaptive backstepping output feedback control scheme for nonlinear FOS was presented via a state

(c) The Author(s) 2019. This article is distributed under the terms of the Creative Commons Attribution 4.0 International License (http://creativecommons.org/licenses/by/4.0/), which permits unrestricted use, distribution, and reproduction in any medium, provided you give appropriate credit to the original author(s) and the source, provide a link to the Creative Commons license, and indicate if changes were made. 
estimation filter and the indirect Lyapunov method in [23]. A fractional order adaptive backstepping control scheme was presented for an incommensurate FOS in the presence of input saturation in [24].

FOS in the triangular form plays an important role in describing many complicated physical phenomena, especially in modeling the circuitry system [28-30]. Recently, the static gain control method has been introduced to study the feedback control design problem of FOS especially fractional-order triangular systems; see [25-27]. Both the state feedback stabilizing controller (SFSC) and the OFSC were designed for both lower triangular and upper triangular linear FOS in [25]. Using the static gain control method and the fractional indirect Lyapunov method, design problems of both the SFSC and the OFSC for the nonlinear lower triangular FOS were investigated in [26]. The SFSC for the NUTFOS was designed in [27].

Based on the above discussions, fewer works have been done to investigate the OFSC for the NUTFOS. In this paper, we study the design of the OFSC for the NUTFOS. The main contributions are as follows:

- Via the fractional indirect Lyapunov method and the static gain control method, the OFSC which makes the closed-loop system asymptotically stable is designed.

- A novel algorithm of designing OFSC for the NUTFOS is established.

- The OFSC for the NUTFOS is linear. The conditions for the existence of the OFSC are very easy to verify.

- The design scheme in this paper is simple because most of works in the design procedure of this paper can be completed by using MATLAB toolbox.

The rest of this paper is organized as follows. Section 2 presents some necessary preliminaries. Section 3 introduces the NUTFOS, investigates the design of the OFSC for the NUTFOS, and establishes an algorithm to design OFSC for the NUTFOS. Section 4 gives an illustrative example to verify our simple method, which is followed by the conclusion in Sect. 5.

\section{Preliminaries}

In this section, we first give some basic preliminaries. Throughout this paper, $D_{t}^{\alpha}$ denotes Caputo fractional derivative, which is referred in $[2,26,31]$.

Definition 2.1 ([2]) Let $h(t)$ be a continuous function. Then $\alpha>0$-order Caputo fractional derivative of $h(t)$ is given by

$$
D_{t}^{\alpha} h(t)=\frac{1}{\Gamma(n-\alpha)} \int_{0}^{t} \frac{h^{(n)}(s)}{(t-s)^{\alpha-n+1}} d s .
$$

Remark 2.1 The Leibniz rule of Caputo fractional derivative is given in [2]. However, it implies that the form of the Leibniz rule of classical derivative is not appropriate for Caputo fractional derivative.

Several fundamental conclusions of Caputo fractional derivative are given in the following. 
Lemma 2.1 ([26]) Let $s_{i}(t)(i=1,2, \ldots, n)$ be continuous and derivable functions and $s(t)=$ $\left(s_{1}(t), s_{2}(t), \ldots, s_{n}(t)\right)^{T} \in \mathbb{R}^{n}$. Then, for any $t \geq 0$,

$$
\frac{1}{2} D_{t}^{\alpha}\left(s^{T}(t) P s(t)\right) \leq s^{T}(t) P D_{t}^{\alpha} s(t)
$$

holds, where $\alpha \in(0,1]$ and $P \in \mathbb{R}^{n \times n}$ is a positive definite matrix.

Remark 2.2 ([26]) Inequality (1) is equivalent to

$$
D_{t}^{\alpha}\left(s^{T}(t) P s(t)\right) \leq\left(D_{t}^{\alpha} s(t)\right)^{T} P s(t)+s^{T}(t) P D_{t}^{\alpha} s(t) .
$$

Finally, we recall the fractional indirect Lyapunov theorem for FOS [31].

Lemma 2.2 ([31]) Let $s=0$ be an equilibrium point of the nonautonomous FOS

$$
D_{t}^{\alpha} s(t)=f(t, s), \quad s_{0} \in \mathbb{R}^{n},
$$

where $0<\alpha<1$. Assume that there exist a Lyapunov function $V(t, v(t))$ and class- $\mathcal{K}$ functions $\beta_{1}, \beta_{2}$, and $\beta_{3}$ satisfying

$$
\begin{aligned}
& \beta_{1}(\|s\|) \leq V(t, s(t)) \leq \beta_{2}(\|s\|), \\
& D_{t}^{\alpha} V(t, s(t)) \leq-\beta_{3}(\|s\|) .
\end{aligned}
$$

Then system (3) is asymptotically stable.

\section{Main results}

In this section, the OFSC for the NUTFOS is designed. Firstly, we introduce the NUTFOS. Then, we investigate the design of the OFSC for the systems by the fractional indirect Lyapunov method and the static gain control method and establish an algorithm to design the OFSC for the systems.

\subsection{Problem description}

In this subsection, the NUTFOS are presented.

Consider the following NUTFOS:

$$
\left\{\begin{array}{l}
D_{t}^{\alpha} v_{1}(t)=v_{2}(t)+g_{1}(t, v(t)), \\
D_{t}^{\alpha} v_{2}(t)=v_{3}(t)+g_{2}(t, v(t)), \\
\vdots \\
D_{t}^{\alpha} v_{n-2}(t)=v_{n-1}(t)+g_{n-2}(t, v(t)), \\
D_{t}^{\alpha} v_{n-1}(t)=v_{n}(t), \\
D_{t}^{\alpha} v_{n}(t)=u(t), \\
y=v_{1}(t),
\end{array}\right.
$$

where $\alpha \in(0,1], v(t)=\left(v_{1}(t), v_{2}(t), \ldots, v_{n}(t)\right)^{T} \in \mathbb{R}^{n}$ denotes the state, $u \in \mathbb{R}$ denotes the input, and $y \in \mathbb{R}$ denotes the output. In this paper, $v_{i}, w_{i}, \bar{e}_{i}$, and $\bar{w}_{i}$ denote $v_{i}(t), w_{i}(t), \bar{e}_{i}(t)$, 
and $\bar{w}_{i}(t)$, respectively. The functions $g_{i}: \mathbb{R} \times \mathbb{R}^{n} \rightarrow \mathbb{R}(i=1,2, \ldots, n-2)$ are continuous and satisfy the following.

\section{Assumption 3.1}

$$
\left|g_{i}(t, v)\right| \leq c\left(\left|v_{i+2}\right|+\left|v_{i+3}\right|+\cdots+\left|v_{n}\right|\right), \quad i=1,2, \ldots, n-2,
$$

where $c \geq 0$.

\subsection{OFSC design}

In this subsection, the design of the OFSC for system (4) is given in terms of the fractional indirect Lyapunov method and the static gain control method, and an algorithm to the proposed method is examined.

Firstly, we present the design of the OFSC for system (4).

Theorem 3.1 Under Assumption 3.1, system (4) is asymptotically stabilized by a linear OFSC.

Proof Examine the following linear observer:

$$
\left\{\begin{array}{l}
D_{t}^{\alpha} w_{1}=w_{2}+\frac{a_{1} l}{R}\left(v_{1}-w_{1}\right), \\
D_{t}^{\alpha} w_{2}=w_{3}+\frac{a_{2} l^{2}}{R^{2}}\left(v_{1}-w_{1}\right), \\
\vdots \\
D_{t}^{\alpha} w_{n-1}=w_{n}+\frac{a_{n-1} l^{n-1}}{R^{n-1}}\left(v_{1}-w_{1}\right), \\
D_{t}^{\alpha} w_{n}=u+\frac{a_{n} l^{n}}{R^{n}}\left(v_{1}-w_{1}\right),
\end{array}\right.
$$

where $R>1, l>0$, and $a_{j}>0(j=1,2, \ldots, n)$ are coefficients of the Hurwitz polynomial

$$
\bar{p}(k)=k^{n}+a_{1} k^{n-1}+\cdots+a_{n-1} k+a_{n}
$$

Set

$$
h_{i}=\frac{v_{i}-w_{i}}{R^{n+1-i}}, \quad \bar{w}_{i}=\frac{w_{i}}{R^{n+1-i}}, \quad i=1,2, \ldots, n
$$

By (4) and (6), we obtain

$$
\begin{aligned}
& D_{t}^{\alpha} h=\frac{1}{R} \bar{A}(l) h+G, \\
& D_{t}^{\alpha} \bar{w}=\frac{1}{R} \bar{\Omega} \bar{w}+\frac{1}{R} F u+\frac{1}{R} \bar{C}(l) h,
\end{aligned}
$$

where

$$
h=\left(\begin{array}{c}
h_{1} \\
h_{2} \\
\vdots \\
h_{n}
\end{array}\right), \quad \bar{A}(l)=\left(\begin{array}{ccccc}
-a_{1} l & 1 & 0 & \cdots & 0 \\
-a_{2} l^{2} & 0 & 1 & \cdots & 0 \\
\vdots & \vdots & \vdots & \ddots & \vdots \\
-a_{n-1} l^{n-1} & 0 & 0 & \cdots & 1 \\
-a_{n} l^{n} & 0 & 0 & \cdots & 0
\end{array}\right) \text {, }
$$




$$
\begin{aligned}
& G=\left(\begin{array}{c}
\frac{g_{1}}{R^{n}} \\
\frac{g_{2}}{R^{n-1}} \\
\vdots \\
\frac{g_{n-2}}{R^{3}} \\
0 \\
0
\end{array}\right), \quad \bar{w}=\left(\begin{array}{c}
\bar{w}_{1} \\
\bar{w}_{2} \\
\vdots \\
\bar{w}_{n}
\end{array}\right), \\
& \bar{\Omega}=\left(\begin{array}{ccccc}
0 & 1 & 0 & \cdots & 0 \\
0 & 0 & 1 & \cdots & 0 \\
\vdots & \vdots & \vdots & \ddots & \vdots \\
0 & 0 & 0 & \cdots & 1 \\
0 & 0 & 0 & \cdots & 0
\end{array}\right), \quad F=\left(\begin{array}{c}
0 \\
0 \\
\vdots \\
0 \\
1
\end{array}\right), \\
& \bar{C}(l)=\left(\begin{array}{cccccc}
a_{1} l & 0 & 0 & \cdots & 0 \\
a_{2} l^{2} & 0 & 0 & \cdots & 0 \\
\vdots & \vdots & \vdots & \ddots & \vdots \\
a_{n-1} l^{n-1} & 0 & 0 & \cdots & 0 \\
a_{n} l^{n} & 0 & 0 & \cdots & 0
\end{array}\right) .
\end{aligned}
$$

We set $R>1$ such that the system consisting of (7), (8) and

$$
u=-\left(b_{1} \bar{w}_{1}+b_{2} \bar{w}_{2}+b_{3} \bar{w}_{3}+\cdots+b_{n} \bar{w}_{n}\right)
$$

is asymptotically stable at $h=0$ and $\bar{w}=0$, where $b_{j}>0(j=1,2, \ldots, n)$ are the coefficients of the Hurwitz polynomial

$$
\bar{q}(k)=k^{n}+b_{n} k^{n-1}+\cdots+b_{2} k+b_{1} .
$$

In forms of $\bar{w}_{i}$ and (9), we obtain

$$
u=-\frac{1}{R^{n}}\left(b_{1} w_{1}+b_{2} R w_{2}+b_{3} R^{2} w_{3}+\cdots+b_{n} R^{n-1} w_{n}\right) .
$$

By (8) and (9), we have

$$
D_{t}^{\alpha} \bar{w}=\frac{1}{R} \bar{B} \bar{w}+\frac{1}{R} \bar{C}(l) h,
$$

where

$$
\bar{B}=\left(\begin{array}{ccccc}
0 & 1 & 0 & \cdots & 0 \\
0 & 0 & 1 & \cdots & 0 \\
\vdots & \vdots & \vdots & \ddots & \vdots \\
0 & 0 & 0 & \cdots & 1 \\
-b_{1} & -b_{2} & -b_{3} & \cdots & -b_{n}
\end{array}\right) .
$$


Let Lyapunov function $\bar{V}_{1}=h^{T} \bar{P}(l) h$, where $\bar{P}(l)>0$ is a positive definite matrix and satisfies $\bar{P}(l) \bar{A}(l)+\bar{A}^{T}(l) \bar{P}(l)=-I$. Then we have

$$
\begin{aligned}
\left.D_{t}^{\alpha} \bar{V}_{1}\right|_{(7)} & \leq\left(D_{t}^{\alpha} h\right)^{T} \bar{P}(l) h+h^{T} \bar{P}(l) D_{t}^{\alpha} h \\
& =\left(\frac{1}{R} \bar{A}(l) h+G\right)^{T} \bar{P}(l) h+h^{T} \bar{P}(l)\left(\frac{1}{R} \bar{A}(l) h+G\right) \\
& \leq-\frac{1}{R}\|h\|^{2}+2\|\bar{P}(l)\| \cdot\|h\| \cdot\|G\| .
\end{aligned}
$$

From (5) and the expressions of $h_{i}$ and $\bar{w}_{i}$, for any $i(i=1,2, \ldots, n)$, we have

$$
\begin{aligned}
\left|\frac{g_{i}}{R^{n-i+1}}\right| & \leq \frac{c}{R^{n-i+1}}\left(\left|v_{i+2}\right|+\left|v_{i+3}\right|+\cdots+\left|v_{n}\right|\right) \\
& \leq \frac{c}{R^{2}} \sum_{j=1}^{n} \frac{\left|v_{j}\right|}{R^{n-j+1}} \\
& =\frac{c}{R^{2}} \sum_{j=1}^{n}\left(\left|h_{j}\right|+\left|\bar{w}_{j}\right|\right) \\
& \leq \frac{c \sqrt{n}}{R^{2}}\|h\|+\frac{c \sqrt{n}}{R^{2}}\|\bar{w}\|,
\end{aligned}
$$

where $R>1$ and $\sum_{j=1}^{n}\left|h_{j}\right| \leq \sqrt{n}\|h\|$.

Hence, we get

$$
\begin{aligned}
\left.D_{t}^{\alpha} \bar{V}_{1}\right|_{(7)} & \leq-\frac{1}{R}\|h\|^{2}+2\|\bar{P}(l)\| \cdot\|h\|\left(\frac{c \sqrt{n}}{R^{2}}\|h\|+\frac{c \sqrt{n}}{R^{2}}\|\bar{w}\|\right)\left\|\left(\begin{array}{c}
1 \\
1 \\
\vdots \\
1 \\
0 \\
0
\end{array}\right)\right\| \\
& \leq-\frac{1}{R}\|h\|^{2}+\frac{2 n c}{R^{2}}\|\bar{P}(l)\| \cdot\|h\|^{2}+\frac{n c}{R^{2}}\|\bar{P}(l)\|\left(\|h\|^{2}+\|\bar{w}\|^{2}\right) \\
& \leq-\frac{1}{R}\|h\|^{2}+\frac{3 n c}{R^{2}}\|\bar{P}(l)\| \cdot\|h\|^{2}+\frac{n c}{R^{2}}\|\bar{P}(l)\| \cdot\|\bar{w}\|^{2} .
\end{aligned}
$$

Set Lyapunov function $\bar{V}_{2}=\bar{w}^{T} \bar{Q} \bar{w}$, where $\bar{Q}>0$ is a positive definite matrix $\bar{Q}>0$ and satisfies $\bar{Q} \bar{B}+\bar{B}^{T} \bar{Q}=-I$. Then we have

$$
\begin{aligned}
\left.D_{t}^{\alpha} \bar{V}_{2}\right|_{(11)} & \leq\left(D_{t}^{\alpha} \bar{w}\right)^{T} \bar{Q} \bar{w}+\bar{w}^{T} \bar{Q} D_{t}^{\alpha} \bar{w} \\
& =\left(\frac{1}{R} \bar{B} \bar{w}+\frac{1}{R} \bar{C}(l) h\right)^{T} \bar{Q} \bar{w}+\bar{w}^{T} \bar{Q}\left(\frac{1}{R} \bar{B} \bar{w}+\frac{1}{R} \bar{C}(l) h\right) \\
& \leq-\frac{1}{R}\|\bar{w}\|^{2}+\frac{2}{R}\|\bar{Q} \bar{C}(l)\| \cdot\|h\| \cdot\|\bar{w}\| \\
& \leq-\frac{1}{R}\|\bar{w}\|^{2}+\frac{1}{R}\|\bar{Q} \bar{C}(l)\|\left(\|h\|^{2}+\|\bar{w}\|^{2}\right) .
\end{aligned}
$$


Choose Lyapunov function $\bar{V}=\bar{V}_{1}+\bar{V}_{2}$. By (12) and (13), we obtain

$$
\begin{aligned}
\left.D_{t}^{\alpha} \bar{V}\right|_{(7)(11)} \leq & -\frac{1}{R}(1-\|\bar{Q} \bar{C}(l)\|)\|h\|^{2}+\frac{3 n c}{R^{2}}\|\bar{P}(l)\| \cdot\|h\|^{2} \\
& -\frac{1}{R}(1-\|\bar{Q} \bar{C}(l)\|)\|\bar{w}\|^{2}+\frac{n c}{R^{2}}\|\bar{P}(l)\| \cdot\|\bar{w}\|^{2} \\
= & -\frac{1}{R^{2}}[(1-\|\bar{Q} \bar{C}(l)\|) R-3 n c\|\bar{P}(l)\|]\|h\|^{2} \\
& -\frac{1}{R^{2}}[(1-\|\bar{Q} \bar{C}(l)\|) R-n c\|\bar{P}(l)\|]\|\bar{w}\|^{2} .
\end{aligned}
$$

Set $l>0$ and $R>1$ satisfying

$$
\|\bar{Q} \bar{C}(l)\| \leq \delta, \quad R>\frac{3 n}{1-\delta}(c\|\bar{P}(l)\|+\eta),
$$

where $\delta$ satisfies $0<\delta<1$ and $\eta>0$.

Thus, from Lemma 2.2 , we get $\left.D_{t}^{\alpha} \bar{V}\right|_{(7)(11)}<-\frac{\eta}{R^{2}}\left(\|h\|^{2}+\|\bar{w}\|^{2}\right)$, which implies that system (7) and (11) is asymptotically stable at $h=0$ and $\bar{w}=0$. Therefore, closed-loop system (4), (8), and (9) is asymptotically stable at $v=0$ and $\bar{w}=0$. Closed-loop system (4), (6), and (10) is also asymptotically stable at $v=0$ and $w=0$. Thus, it can be concluded that system (6) and (10) is the linear output dynamic compensator of system (4). This completes the proof.

Based on the above analysis, we establish an algorithm to construct an OFSC for system (4).

Algorithm 3.1 The algorithm is divided into the following five steps:

(1) Let $a_{j}>0, b_{j}>0(j=1,2, \ldots, n)$ be the coefficients of the Hurwitz polynomials

$$
\begin{aligned}
& \bar{p}(k)=k^{n}+a_{1} k^{n-1}+\cdots+a_{n-1} k+a_{n}, \\
& \bar{q}(k)=k^{n}+b_{n} k^{n-1}+\cdots+b_{2} k+b_{1} .
\end{aligned}
$$

Then we get $\bar{A}(l), \bar{C}(l)$, and $\bar{B}$.

(2) Solving the equation

$$
\bar{B}^{T} \bar{Q}+\bar{Q} \bar{B}=-I
$$

leads to $\bar{Q}>0$.

(3) Choose an appropriate constant $l$ such that $\delta=\|\bar{Q} \bar{C}(l)\|<1$.

(4) Solve the equation

$$
\bar{P}(l) \bar{A}(l)+\bar{A}^{T}(l) \bar{P}(l)=-I .
$$

Then we obtain $\bar{P}(l)>0$.

(5) Let

$$
R>\frac{3 n}{1-\delta} c\|\bar{P}(l)\|
$$


Then a linear OFSC for system (4) is $u$, where $u$ is defined as in (10), and $w_{1}, w_{2}, \ldots, w_{n}$ are the states of system (6).

Remark 3.1 The different research problems of this paper and [27] are on the design problem of the OFSC output and the SFSC for the same system (4). The OFSC for the NUTFOS is studied in this paper, and the SFSC for the NUTFOS is considered in [27]. In fact, the design process of the OFSC is more complicated than that of the SFSC.

\section{An example}

In this section, we give an example to verify our simple method.

Example 4.1 Consider the following nonlinear FOS:

$$
\left\{\begin{array}{l}
D_{t}^{\alpha} v_{1}=v_{2}-\frac{\sin v_{1}}{c+c e^{-t}} v_{3} \\
D_{t}^{\alpha} v_{2}=v_{3} \\
D_{t}^{\alpha} \nu_{3}=u \\
y=v_{1}
\end{array}\right.
$$

where $\alpha \in(0,1]$.

By Algorithm 3.1, choose $a_{1}=99 / 100, a_{2}=261 / 1000, a_{3}=81 / 5000, b_{1}=100, b_{2}=120$, $b_{3}=21$. Then

$$
\begin{aligned}
& \bar{A}(l)=\left(\begin{array}{ccc}
-99 l / 100 & 1 & 0 \\
-261 l^{2} / 1000 & 0 & 1 \\
-81 l^{3} / 5000 & 0 & 0
\end{array}\right), \quad \bar{C}(l)=\left(\begin{array}{ccc}
99 l / 100 & 0 & 0 \\
261 l^{2} / 1000 & 0 & 0 \\
81 l^{3} / 5000 & 0 & 0
\end{array}\right), \\
& \bar{B}=\left(\begin{array}{ccc}
0 & 1 & 0 \\
0 & 0 & 1 \\
-100 & -120 & -21
\end{array}\right), \quad \bar{Q}=\left(\begin{array}{ccc}
4 & -1 / 2 & -1 \\
-1 / 2 & 1 & -1 / 2 \\
-1 & -1 / 2 & 1
\end{array}\right) \text {. }
\end{aligned}
$$

Set $l=0.9$ and $c=3000$. Then, we get $R=6.5$. Therefore, the linear output feedback stabilizer for system (14) is

$$
u=-\frac{100}{R^{3}} w_{1}-\frac{120}{R^{2}} w_{2}-\frac{21}{R} w_{3}
$$

where $w_{1}, w_{2}$, and $w_{3}$ are the states of the following system:

$$
\left\{\begin{array}{l}
D_{t}^{\alpha} w_{1}=w_{2}+\frac{99 l}{100 R}\left(v_{1}-w_{1}\right), \\
D_{t}^{\alpha} w_{2}=w_{3}+\frac{261 l^{2}}{100 R^{2}}\left(v_{1}-w_{1}\right), \\
D_{t}^{\alpha} w_{3}=-\frac{100}{R^{3}} w_{1}-\frac{120}{R^{2}} w_{2}-\frac{21}{R} w_{3}+\frac{81 l^{3}}{5000 R^{3}}\left(v_{1}-w_{1}\right) .
\end{array}\right.
$$

Figures 1, 2, and 3 show the state trajectories of the system consisting of (14), (15), and (16) with the order $\alpha=0.9$ for the initial condition $\left(v_{1}(0), v_{2}(0), v_{3}(0), w_{1}(0), w_{2}(0), w_{3}(0)\right)=$ $(-0.03,0.2,0.6,0.1,0.5,0.04)$, which shows the asymptotic stability of the system. 


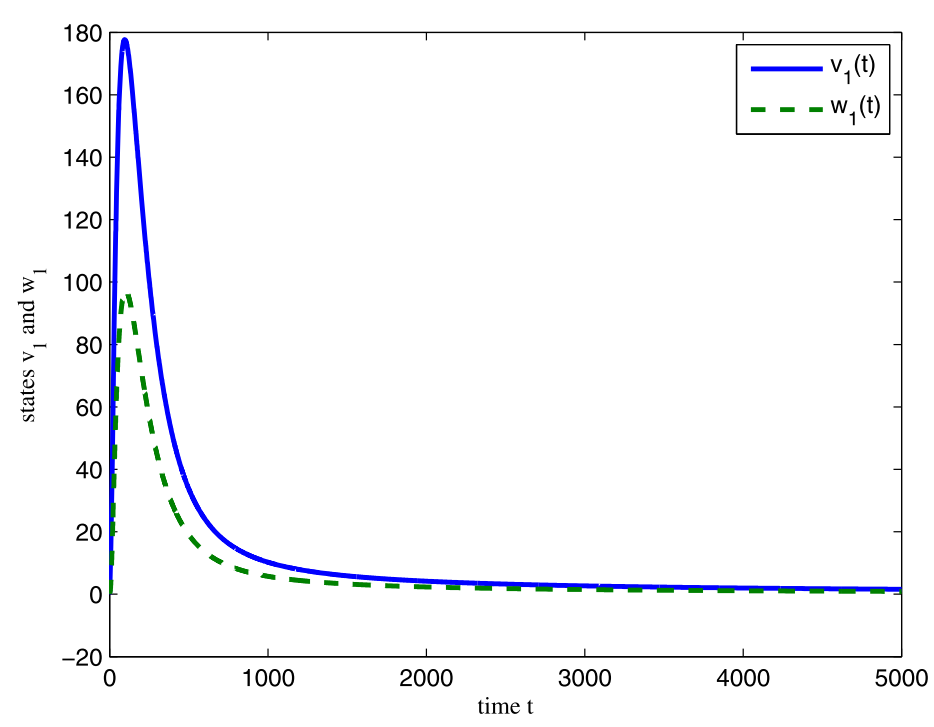

Figure 1 Trajectories of $v_{1}$ and $w_{1}$

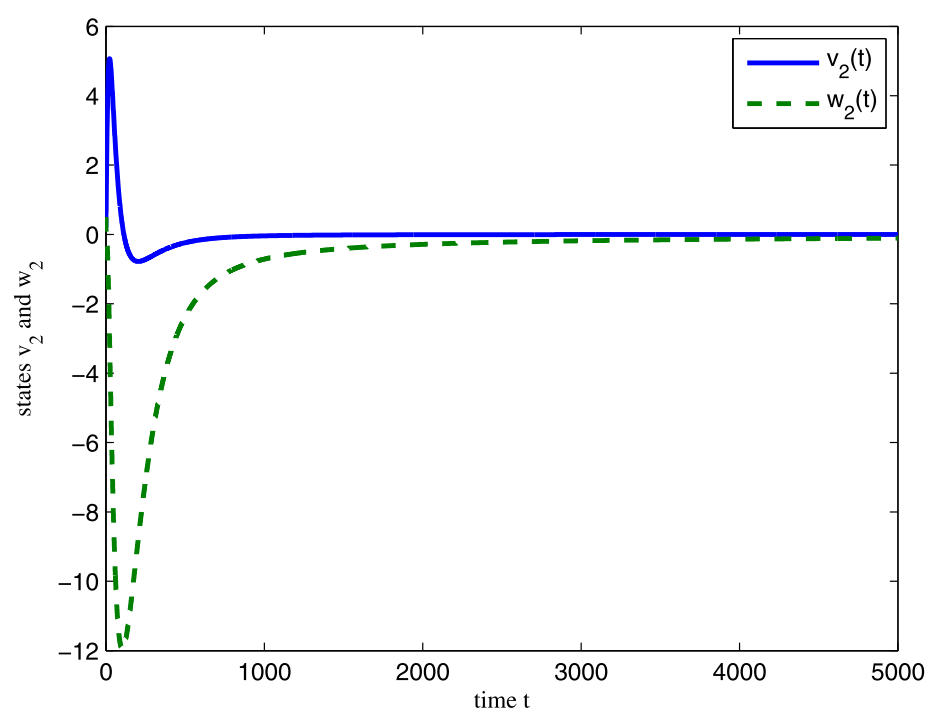

Figure 2 Trajectories of $v_{2}$ and $w_{2}$

\section{Conclusion}

By using the fractional indirect Lyapunov method and the static gain control method, a simple method of design problem of the OFSC for the NUTFOS has been investigated in this paper. We have obtained the OFSC making the closed-loop system asymptotically stable and established an algorithm to design output stabilizing controller for the NUTFOS. Finally, an example has been given to verify the validity of the simple method.

In future works, one can study the controllability of the nonlinear upper triangular fractional-order systems. 


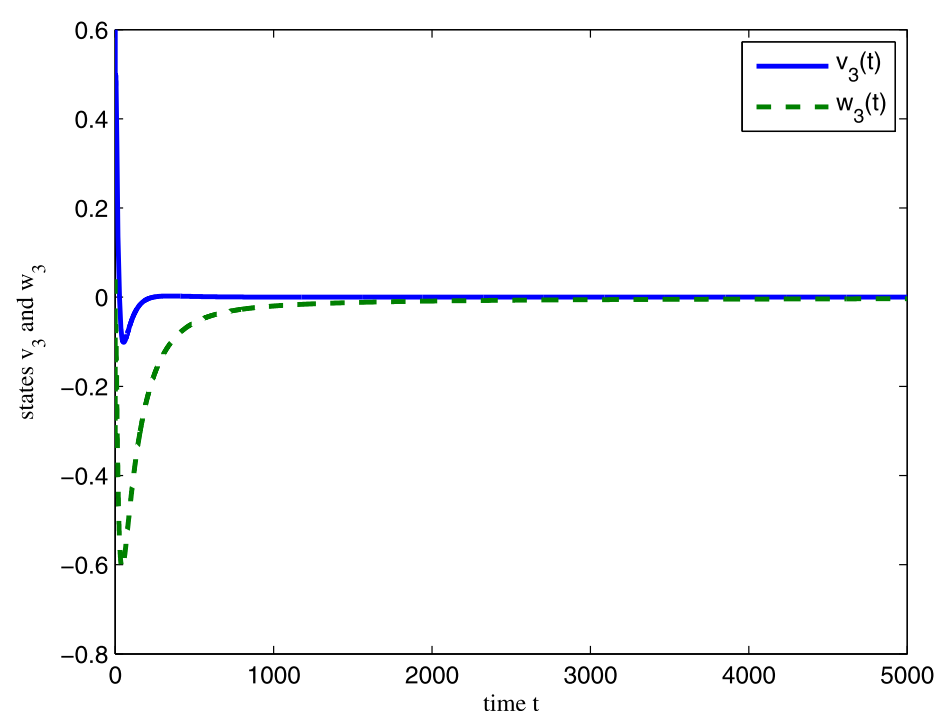

Figure 3 Trajectories of $v_{3}$ and $w_{3}$

\section{Acknowledgements}

The authors sincerely thank the reviewers for their valuable suggestions and useful comments that have led to the present improved version of the original manuscript.

\section{Funding}

This research is supported by the National Natural Science Foundation of China $(61703180,61803176,61877028$, 61807015, 61773010), the Natural Science Foundation of Shandong Province (ZR2017BA010, ZR2017LF012), the Project of Shandong Province Higher Educational Science and Technology Program (J18KA230, J17KA157), and the Scientific Research Foundation of University of Jinan $(1008399,160100101)$.

\section{Competing interests}

The authors declare that they have no competing interests.

\section{Authors' contributions}

The authors declare that the study was realized in collaboration with the same responsibility. All authors read and approved the final manuscript.

\section{Author details}

'School of Mathematical Sciences, University of Jinan, Jinan, P.R. China. '2Business School, University of Jinan, Jinan, P.R. China. ${ }^{3}$ College of Mathematics and System Science, Shandong University of Science and Technology, Qingdao, P.R. China.

\section{Publisher's Note}

Springer Nature remains neutral with regard to jurisdictional claims in published maps and institutional affiliations.

Received: 27 February 2019 Accepted: 14 April 2019 Published online: 25 April 2019

\section{References}

1. Podlubny, l.: Fractional Differential Equations. Academic Press, Cambridge (1999)

2. Kilbas, A.A., Srivastava, H.H., Trujillo, J.J.:Theory and Applications of Fractional Differential Equations. Elsevier, Amsterdam (2006)

3. Wu, G., Baleanu, D., Luo, W.: Lyapunov functions for Riemann-Liouville-like fractional difference equations. Appl. Math. Comput. 314, 228-236 (2017)

4. Wei, Y., Song, Q., Bai, Z:: Existence and iterative method for some fourth order nonlinear boundary value problems Appl. Math. Lett. 87, 101-107 (2019)

5. Wang, Z., Xie, Y., Lu, J., et al.: Stability and bifurcation of a delayed generalized fractional-order prey-predator model with interspecific competition. Appl. Math. Comput. 347, 360-369 (2019)

6. Chatzarakis, G.E., Li, T.: Oscillation criteria for delay and advanced differential equations with nonmonotone arguments. Complexity 2018, Article ID 8237634 (2018)

7. Jiang, C., Zhang, F., Li, T.: Synchronization and antisynchronization of $\mathrm{N}$-coupled fractional-order complex chaotic systems with ring connection. Math. Methods Appl. Sci. 41, 2625-2638 (2018)

8. Li, T., Rogovchenko, Y.V:: Oscillation criteria for second-order superlinear Emden-Fowler neutral differential equations. Monatshefte Math. 184, 489-500 (2017) 
9. Zhao, Y., Hou, X., Sun, Y., et al.: Solvability for some class of multi-order nonlinear fractional systems. Adv. Differ. Equ. 2019, Article ID 23 (2019)

10. Zhao, Y.: Solvability for nonlinear singular fractional differential systems with multi-orders. J. Appl. Anal. Comput. 8, 1170-1185 (2018)

11. Zhao, Y., Sun, S., Han, Z., et al.: Positive solutions for boundary value problems of nonlinear fractional differential equations. Appl. Math. Comput. 217, 6950-6958 (2011)

12. Song, Q., Bai, Z:: Positive solutions of fractional differential equations involving the Riemann-Stieltjes integral boundary condition. Adv. Differ. Equ. 2018, Article ID 183 (2018)

13. Sheng, K., Zhang, W., Bai, Z.: Positive solutions to fractional boundary value problems with $p$-Laplacian on time scales. Bound. Value Probl. 2018, Article ID 70 (2018)

14. Bai, Z., Chen, Y., Lian, H., et al.: On the existence of blow up solutions for a class of fractional differential equations. Fract. Calc. Appl. Anal. 17, 1175-1187 (2014)

15. Li, S.: Robust stability and stabilization of LTI fractional-order systems with poly-topic and two-norm bounded uncertainties. Adv. Differ. Equ. 2018, Article ID 88 (2018)

16. Xu, Q., Zhuang, S., Xu, X., et al.: Stabilization of a class of fractional-order nonautonomous systems using quadratic Lyapunov functions. Adv. Differ. Equ. 2018, Article ID 14 (2018)

17. Ma, Y., Lu, J., Chen, W.: Robust stability and stabilization of fractional order linear systems with positive real uncertainty. ISA Trans. 53, 199-209 (2014)

18. Ji, Y., Su, L., Qiu, J.: Design of fuzzy output feedback stabilization for uncertain fractional-order systems. Neurocomputing 173, 1683-1693 (2016)

19. Wei, Y., Peter, W.T., Yao, Z., et al.: The output feedback control synthesis for a class of singular fractional order systems. ISA Trans. 69, 1-9 (2017)

20. Zhan, T., Liu, X., Ma, S.: A new singular system approach to output feedback sliding mode control for fractional order nonlinear systems. J. Franklin Inst. 355, 6746-6762 (2018)

21. Badri, P., Sojoodi, M.: Robust fixed-order dynamic output feedback controller design for fractional-order systems. IET Control Theory Appl. 12, 1236-1243 (2017)

22. Ding, D., Qi, D., Wang, Q.: Non-linear Mittag-Leffler stabilisation of commensurate fractional-order non-linear systems. IET Control Theory Appl. 9, 681-690 (2015)

23. Wei, Y., Peter, W.T., Yao, Z., et al.: Adaptive backstepping output feedback control for a class of nonlinear fractional order systems. Nonlinear Dyn. 86, 1047-1056 (2016)

24. Sheng, D., Wei, Y., Cheng, S., et al.: Adaptive backstepping control for fractional order systems with input saturation. J. Franklin Inst. 354, 2245-2268 (2017)

25. Zhang, X., Liu, L., Feng, G., et al.: Asymptotical stabilization of fractional-order linear systems in triangular form. Automatica 49, 3315-3321 (2013)

26. Zhao, Y., Wang, Y., Zhang, X., et al.: Feedback stabilisation control design for fractional order non-linear systems in the lower triangular form. IET Control Theory Appl. 10, 1061-1068 (2016)

27. Zhao, Y., Wang, Y., Li, H.: State feedback control for a class of fractional order nonlinear systems. IEEE/CAA J. Autom. Sin. 3, 483-488 (2016)

28. Ahmad, W.M., El-Khazali, R., Al-Assaf, Y.: Stabilization of generalized fractional order chaotic systems using state feedback control. Chaos Solitons Fractals 22, 141-150 (2004)

29. Petras, I.: Fractional-Order Nonlinear Systems. Springer, Berlin (2011)

30. Kaczorek, T.: Selected Problems of Fractional Systems Theory. Springer, Berlin (2011)

31. Li, Y., Chen, Y., Podlubny, I.: Mittag-Leffler stability of fractional order nonlinear dynamic systems. Automatica 45, 1965-1969 (2009)

\section{Submit your manuscript to a SpringerOpen ${ }^{\circ}$ journal and benefit from:}

- Convenient online submission

- Rigorous peer review

- Open access: articles freely available online

- High visibility within the field

- Retaining the copyright to your article

Submit your next manuscript at $\gg$ springeropen.com 